\title{
On the time-varying trend in global-mean surface temperature
}

\author{
Zhaohua Wu $\cdot$ Norden E. Huang $\cdot$ John M. Wallace $\cdot$ \\ Brian V. Smoliak $\cdot$ Xianyao Chen
}

Received: 19 January 2010/ Accepted: 17 June 2011/Published online: 7 July 2011

(C) Springer-Verlag 2011

\begin{abstract}
The Earth has warmed at an unprecedented pace in the decades of the 1980s and 1990s (IPCC in Climate change 2007: the scientific basis, Cambridge University Press, Cambridge, 2007). In Wu et al. (Proc Natl Acad Sci USA 104:14889-14894, 2007) we showed that the rapidity of the warming in the late twentieth century was a result of concurrence of a secular warming trend and the warming phase of a multidecadal ( $~ 65$-year period) oscillatory variation and we estimated the contribution of the former to be about $0.08^{\circ} \mathrm{C}$ per decade since $\sim 1980$. Here we demonstrate the robustness of those results and discuss their physical links, considering in particular the shape of the secular trend and the spatial patterns associated with the secular trend and the multidecadal variability. The shape of the secular trend and rather globally-uniform spatial pattern associated with it are both suggestive of a response to the buildup of well-mixed greenhouse gases.
\end{abstract}

\section{Z. Wu}

Department of Meteorology and Center for Ocean-Atmospheric Prediction Studies, Florida State University,

Tallahassee, FL, USA

N. E. Huang $(\bowtie)$

Research Center for Adaptive Data Analysis Center, National Central University, Chungli 32001, Taiwan

e-mail: norden@ncu.edu.tw

J. M. Wallace · B. V. Smoliak

Department of Atmospheric Sciences, University of Washington, Seattle, WA, USA

X. Chen

The First Institute of Oceanography,

State Oceanic Administration, Qingdao, China
In contrast, the multidecadal variability tends to be concentrated over the extratropical Northern Hemisphere and particularly over the North Atlantic, suggestive of a possible link to low frequency variations in the strength of the thermohaline circulation. Depending upon the assumed importance of the contributions of ocean dynamics and the time-varying aerosol emissions to the observed trends in global-mean surface temperature, we estimate that up to one third of the late twentieth century warming could have been a consequence of natural variability.

Keywords Global warming trend - Multidecadal variability · Ensemble empirical mode decomposition . IPCC AR4

\section{Introduction}

The time series of observation-based global-mean surface temperature (GST) has been a focal point for investigations of human-induced global warming. Of particular interest is the estimation and attribution of the secular trend (ST). Four different estimates of linear trends of observationbased GST presented in Figure TS.6 of the Technical Summary of the Fourth Assessment Report (AR4) of the Intergovernmental Panel on Climate Change (IPCC 2007) fitted for different timescales, ranging from a warming trend of $0.045 \pm 0.012^{\circ} \mathrm{C} /$ decade for the past 150 years to $0.177 \pm 0.052^{\circ} \mathrm{C} /$ decade for the most recent 25 years (both periods ended at 2003), are shown in Fig. 1, together with a time series of the 25-year running linear trend. It is apparent from that figure that global warming has proceeded in a stepwise fashion, with relatively rapid rates of temperature increase from 1915 to 1935 and from 1980 to 1998 alternating with periods with much weaker and 


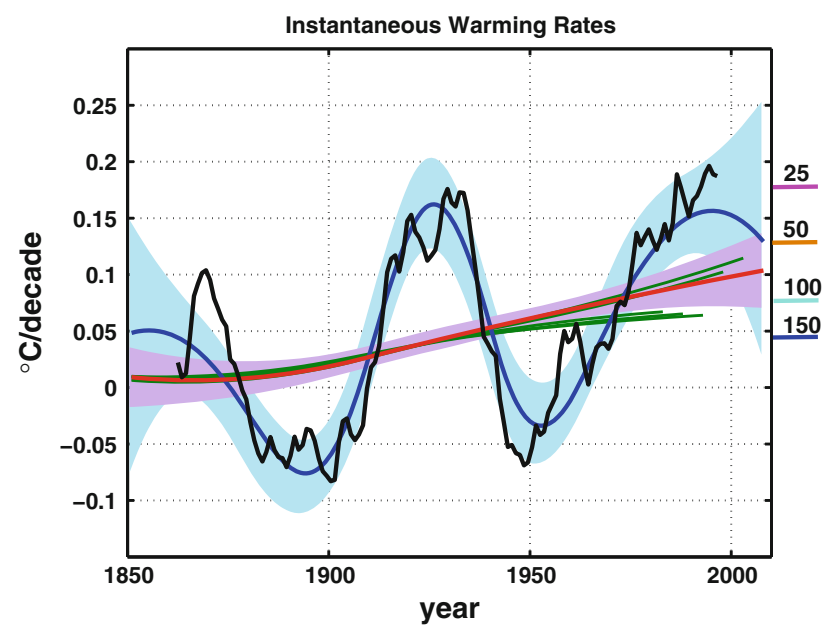

Fig. 1 Global warming rates. The black solid line is the estimated warming rate for the running 25-year linear trend; the red (blue) solid line is the warming rate based on the mean ST (ST + MDV) of the GST for the period 1850-2008 as determined from EEMD. The green lines are the warming rates based on mean STs of GST ending in 1983, 1988, 1993, 1998, and 2003, respectively. The purple area illustrates the confidence intervals (within two standard deviations) for the instantaneous warming rates of ST of 1,000 randomly sampled yearly GSTs for the period 1850-2008. The brown The magenta, brown, cyan, and dark-blue short lines on the right hand side of the figure are the warming rates $\left({ }^{\circ} \mathrm{C}\right.$ per decade) for the past $25,50,100$, and 150 -years as defined by linear trends ending in 2005, as reported in the AR4 of IPCC, respectively

sometimes even negative trends centered around 1900 and $1950 .{ }^{1}$ These statistics serve to illustrate the sensitivity of estimates of such linear trends to the choice of start and end points upon which they are based. Short-term linear trends are an amalgamation of the ST and fluctuations with timescales too long to be resolved by conventional time series analysis techniques. The interpretation of the multidecadal variability (MDV) is particularly problematic in this respect.

Distinguishing between cycles and time varying ST of a time series has long been regarded as a daunting problem, as exemplified by the statement of Stock and Watson (1988): "one economist's 'trend' can be another's 'cycle". The most widely used method of determining the trend in a data set is to draw the least squares best fit straight line within prescribed intervals, as was done in IPCC AR4. In reality, the rate of increase of GST in response to the cumulative buildup of long lived greenhouse gases and the changing rates of emission of aerosols is time dependent. Representing secular trends in GST in terms of linear trends is often not physically realistic. A more informative

\footnotetext{
1 In contrast, the 25-year running linear trend of AR4 multimodel ensemble does not contain stepwise fashion; rather, it varies little for the periods before 1963 or after 1963 which coincided with Agung volcano eruption. The linear trend is about $0.06^{\circ} \mathrm{C} / \mathrm{dec}$ ade for the period $1900-1963$ and about $0.19^{\circ} \mathrm{C} /$ decade for the period after 1963 .
}

representation is an intrinsically-determined monotonic curve, having at most one extremum within a given time span (Huang et al. 1998; Wu et al. 2007).

Using the above definition of the trend is likely to be more true to the observations than fitting data with straight lines within arbitrarily selected time intervals or with other arbitrarily pre-determined curves (e.g., exponential curve, polynomials of various orders). There is no guarantee that the modes recovered using such prescribed analytic functions correspond to physical modes of variability. Hence, it is desirable to have a more objective and non-parametric method of quantifying the low frequency variability of a time series such as GST.

If the cycles and secular trend extracted from the data do reflect the physical processes operating at a given time, then they should be temporally local quantities and the corresponding physical interpretation within specified time intervals should also not change with the addition of new data, for the subsequent evolution of a physical system cannot alter the reality that has already happened. Indeed, temporal locality should be the first principle in guiding all the time series analysis. This requirement reflects the evolution of time series analysis from the Fourier transform, to the windowed Fourier transform (Gabor 1946) and on to wavelet analysis (Daubechies 1992). It can be verified that the linear trends as fitted in AR4 (IPCC 2007) do not satisfy this locality principle, while the adaptive trend defined in Wu et al. (2007) and extracted using the ensemble empirical mode decomposition (EEMD) method (Huang and Wu 2008; Wu and Huang 2009) satisfies it qualitatively at least (as will be shown later), and hence, the ST determined adaptively by the data has a better chance of reflecting the underlying physics and resolving the ambiguity between the trend and the fluctuations superimposed upon it.

In this paper, we will recalculate the ST of GST using EEMD, which is a major refinement of the original Empirical Mode Decomposition (EMD) method used in $\mathrm{Wu}$ et al. (2007). It is expected that the partitioning of a time series into oscillatory variations on various timescales and an ST using an adaptive and temporal local analysis method, such as EEMD, provides an improved means of estimating the global warming trend from a data analysis perspective. In addition, a down-sampling method is devised to estimate the uncertainties of MDV, ST and their instantaneous rates of change. The temporally locality of the extracted modes is examined, with emphasis on the multi-decadal variability and the secular trend. The sensitivity of ST and MDV time series with respect to the different analyses of surface temperature, to the most recent corrections of the surface temperature analysis, to the inclusion or exclusion of the response to volcanic aerosol forcing, and to the presence of noise in the data will be tested. 
A closely related question is the degree to which the MDV and the ST components of GST recovered using EEMD correspond, respectively, to the natural and anthropogenically-forced components of the GST variability. ST obtained from EEMD does not capture any anthopogenically-induced global warming that may be present on multi-decadal or shorter timescales and, conversely, it is conceivable that natural variability could project upon the secular trend. That ST and MDV might nonetheless be useful for representing the anthropogenic and natural components of the decadal variability draws support from recent studies of Semenov et al. (2010) and DelSole et al. (2011). Based on an analysis of numerical experiments with a coupled (atmosphere/ocean) model, Semenov et al. showed that the internal variability of Atlantic Meridional Overturning (AMO) circulation "could have considerably contributed to the Northern Hemisphere surface warming since 1980". By projecting observed SST data onto spatial patterns derived from a statistical analysis of Coupled Model Intercomparison Project (CMIP3) simulations, Delsole et al. were able to formally partition the observed variability in 20th century GST into anthropogenically forced and natural components. They concluded that most of the irregularities in the rate of rise on GST on the multidecadal time scale can be attributed to natural (coupled atmosphere/ocean) variability. That our observational results are in agreement with the results of these studies lend credence to the notion that separating the low frequency variability of GST into ST and MDV components using EEMD series may be useful for attribution.

The paper is arranged as the follows: Sect. 2 introduces data and methods used in this study; Sect. 3 presents the major results: including the partitioning of the secular trend and variability on various timescales, their statistical significance, the temporally local warming rate as inferred from the time derivative of ST, an assessment of the robustness of the ST and MDV modes, the global structures of SST variability and change associated with MDV and ST, as well as the link of MDV to the natural variability of Atlantic Meridional Overturning (AMO) circulation. The final section summarizes the main results and provides some caveats relating to this study and some broader conclusions relating to the role of observational studies in the science of global climate change.

\section{Data and methods}

\subsection{Data}

The data used in this study include

1. Global monthly land and sea surface temperature from HadCRUT3v dataset (Jones et al. 1999; Rayner et al. 2003);
2. Global monthly land and sea surface temperature analyses provided by Goddard Institute for Space Studies (GISTEMP) (Hansen et al. 1999);

3. The surface atmospheric temperature (SAT) dataset, which covers a time span from 1900 to December 2006, from the Global Historical Climatology Network, version 3 (Peterson and Vose 1997). The SST dataset is the NOAA ERSST by Smith et al. (2008);

4. The International Comprehensive Ocean-Atmosphere Data Set (ICOADS), which contains objectively analyzed in-situ observations of SST in $5^{\circ} \times 5^{\circ}$ grid boxes (Smith and Reynolds 2005); and

5. An estimate of the variations in global-mean surface temperature variability attributable to volcanic forcing (Thompson et al. 2009).

\subsection{The ensemble empirical mode decomposition ${ }^{2}$}

To extract trends in real data in accordance with the definition mentioned in the previous section, an adaptive and temporal local analysis method, the recently developed ensemble empirical mode decomposition (EEMD) method (Huang and Wu 2008; Wu and Huang 2009) is used.

EEMD is based on EMD (Huang et al. 1998; Huang and Wu 2008), a method that emphasizes the adaptiveness and temporal locality of the data decomposition. Many traditional decomposition methods, including the Fourier Transform and wavelet decomposition methods, utilize a priori determined basis functions, which may faithfully represent the characteristics of a time series in some segments but not in other segments of a non-stationary time series (Härdle 1990; Fan and Yao 2005). Other methods, including empirical decomposition methods that rely heavily on autocorrelations, involve implicitly global temporal domain integrals and therefore, are non-local and not well suited for extracting physically meaningful information from non-stationary time series. EMD, which uses extrema information of the riding waves in non-stationary time series, is an adaptive and temporally local decomposition method that extracts successively the riding amplitude-frequency modulated oscillatory components, starting with the highest frequencies and proceeding toward the lowest frequencies successively without using any a priori determined basis functions.

\subsubsection{The empirical mode decomposition}

EMD was a two-stage adaptive and temporally-local timefrequency analysis algorithm, first developed aiming at providing a more accurate expression of a time series in a

\footnotetext{
2 The Matlab code of EEMD and a simple tutorial for how to use the code can be found in http://www.rcada.ncu.edu.tw/research1.htm.
} 
time-frequency-energy domain (Huang et al. 1998, 1999, 2003, Huang and $\mathrm{Wu} 2008)$. In EMD, the data $x(t)$ are decomposed in terms of "intrinsic mode functions" (IMFs), $c_{j}$, i.e.,

$x(t)=\sum_{j=1}^{n} c_{j}(t)+r_{n}(t)$,

where

$c_{j}(t)=a_{j}(t) \cos \left[\int \omega_{j}(t) d t\right]$,

and $r_{n}$ is the residual of the data $x(t)$, after $n$ intrinsic mode functions (IMFs) have been extracted. In practice, the EMD is implemented through a sifting process that uses only local extrema. For any data set, $x(t)=r_{j-1}$, say, the procedure is as follows: (1) identify all the local extrema (the combination of both maxima and minima) and connect all these local maxima (minima) with a cubic spline as the upper (lower) envelope; (2) obtain the first component $h$ by taking the difference between the data and the local mean of the upper and lower envelopes; and (3) treat $h$ as the data and repeat steps 1 and 2 as many times as is required until the envelopes are symmetric about zero to within a certain tolerance. The final $h$ is designated as $c_{j}$. The sifting process is considered to be complete when the residue, $r_{n}$, becomes a monotonic function or a function containing only one internal extremum from which no more IMFs can be extracted.

From above algorithm description, it is clear that EMD is not a curve fitting method in which an a priori determined functional form is used, for the piece-wise cubic spline fitting between neighboring maxima (minima) is not sensitive to maxima (minima) far away and is thereby quite local. It has also been tested that using a higher order spline instead of a cubic spline would not change the results significantly (Huang and Wu 2008). By applying EMD, the secular trend of a time series is naturally obtained after all the oscillatory components (riding waves) are removed from the time series. Since its development about 10 years ago, EMD has found numerous successful applications in many different scientific and engineering fields and has accumulated thousands of citations.

\subsubsection{Calculation of the instantaneous amplitude and frequency of a component}

After a time series is decomposed into IMFs, natural amplitude-frequency modulated oscillatory functions, various methods can be applied to obtain instantaneous frequencies for each IMF that lead to a time-frequency-energy representation of data. Traditionally, the Hilbert transform (Gabor 1946; Van der Pol 1946) is applied to calculate the accompanying imaginary part of an IMF and obtain the complex expression of an IMF of which the instantaneous amplitude and frequency can be calculated (Huang et al. $1998,1999)$. For any function $c_{j}(t)$, its Hilbert transform $y_{j}(t)$ is

$y_{j}(t)=\frac{1}{\pi} \int_{-\infty}^{\infty} \frac{c_{j}(\tau)}{t-\tau} d \tau$.

With the Hilbert transform $y_{j}(t)$ of the function $c_{j}(t)$, one obtains an analytic function,

$z(t)=c_{j}(t)+i y_{j}(t)=a_{j}(t) e^{i \theta_{j}(t)}$,

where $i=\sqrt{-1}$,

$a_{j}(t)=\left(c_{j}^{2}+y_{j}^{2}\right)^{1 / 2} ; \quad \theta_{j}(t)=\arctan \frac{y_{j}}{c_{j}}$.

Here $a_{j}(t)$ is the instantaneous amplitude, and $\theta_{j}(t)$ is the instantaneous phase function. The instantaneous frequency is simply

$\omega_{j}(t)=\frac{d \theta_{j}(t)}{d t}$.

Equations 1a, 1b, 4a, 4b constitute a time-frequencyenergy distribution of time series $x(t)$.

\subsubsection{The ensemble empirical mode decomposition and the direct quadrature method}

There have been two major subsequent elaborations of the EMD algorithm that have been motivated by practical problems of EMD. The first problem is that the EMD results are unstable with respect to noise of data for noise can alter the distribution of extrema, thereby leading to the lack of robustness of IMFs obtained using EMD. This drawback leads to difficulty in physical interpretation of IMFs. To solve this problem, EEMD was developed (Wu and Huang 2009). In this method, counter-intuitively, multiple noise realizations are added to the unique time series of "observations" $x(t)$ to mimic a scenario of multiple realizations from which an ensemble average approach for the corresponding IMFs can be used to extract scale-consistent signals. The major steps in the EEMD method are as follows: (1) add a white noise series to the targeted data; (2) decompose the data with the added white noise into IMFs; (3) repeat step 1 and 2 again and again, but with different white noise series each time; and (4) obtain (ensemble) means of the respective IMFs of the decompositions as the final result.

From observation and intuition, the effects of the decomposition based on EEMD are quite understandable: the added white noise series cancel each other, and the 
mean IMFs stays within the natural dyadic filter windows as discussed in Flandrin et al. (2004) and Wu and Huang (2004, 2005), significantly improving the dyadic property of the decomposition and leading to stable decompositions. Therefore, this elaboration renders the EMD/EEMD method much more robust, eliminating many side effects formerly caused by unphysical scale mixing due to the presence of noise in the data. This development has also led to the most recent extension to multi-dimensional EEMD (Wu et al. 2009).

The second problem with EMD is associated with using Hilbert transform to calculate the instantaneous frequency. Due to the Hilbert transform being a global domain integral, the instantaneous amplitude and instantaneous frequency obtained using the Hilbert Transform is not "temporally local" or instantaneous. To overcome this problem, the direct quadrature (DQ) algorithm is proposed as a means of obtaining the instantaneous amplitude and instantaneous frequency (Huang et al. 2009a). The principle behind the DQ is very simple: if an IMF $c_{j}(t)$ is obtained, its amplitude $a_{j}(t)$ can be obtained simply by connecting the maxima of $c_{j}(t)$. With known $c_{j}(t)$ and $a_{j}(t)$, using Eq. 1b, one can obtain the instantaneous frequency directly without using the Hilbert transform. It has been verified that DQ provides a more accurate calculation of the instantaneous frequency than the traditional method based on the Hilbert transform (Huang et al. 2009a).

\subsection{Determination of trend uncertainty using down sampling}

One issue associated with EMD/EEMD for determining trends in GST must be discussed here: the so called "data end effect". Any method in current use is subject to uncertainties due to the data end effect. For example, the Fourier transform has the Gibbs effect and the wavelet analysis has its "cone of influence" (Torrence and Compo 1998). For EMD/EEMD, the error related to the data end effect is tied to the determination of values of envelopes at the data ends in every recurrence of the sifting process. When many of the widely used data end treatments in other methods, such as repetitiveness of data (in the Fourier transform) and mirror and anti-mirror extensions (for example, in wavelet analysis) were tested, it was found that EMD has a cone of influence analogous to the one in wavelet analysis but not as serious (Gledhill 2003). This drawback has led us to develop a new data end effect treatment scheme for predicting the values of the ends of the envelopes using information on the nearest two maxima (minima) to a data end for every recursion of the sifting process (Wu et al. 2009). This scheme has been demonstrated to reduce significantly the size of the "cone of influence" in numerous tests with synthetic data and real world data, especially in EEMD in which the added noise perturbation to the data helps to "correct" the predictions of the envelope ends for low frequency components. However, for the case of decomposing GST, the sum of the MDV and ST only has two interior maxima and two interior minima; and the strong amplitude-frequency modulation of the MDV could potentially lead to significant error in the separation of MDV and ST for the linear extension method may not approximate the highly nonlinear amplitude modulation of MDV accurately in this case.

Another related issue is that the noise contained in the data could lead to the errors in the estimated MDV and ST of GST. In general, all data are amalgamations of signal and noise, i.e.,

$x(t)=s(t)+n(t)$

in which $x(t)$ is the recorded data, and $s(t)$ and $n(t)$ are the true signal and noise, respectively. When the noise has a significant portion of its energy at the low frequencies (such as warm color noise), undoubtedly, the determined lower frequency components of the signal will be significantly contaminated by the lower frequency part of noise. Unfortunately, the noise, or even its characteristics, in GST is not a known a priori; and therefore we can not directly separate the noise from the signal. In such a case, if we want to estimate the statistical significance of any component of GST, we need to make a null hypothesis based on our limited understanding of how GST changes, such as in "Appendix" in which a single variable red noise null hypothesis is tested.

To estimate the uncertainties in the determined MDV and ST components of GST in addressing the two issues discussed above, we here devise a down sampling approach that bypasses these two issues. In this new approach, we randomly pick a value of the monthly GST for each calendar year to represent the entire annual average, which leads to a yearly down-sampled GST series. Theoretically, this approach could yield $12^{159}$ different time series. Among them, we randomly selected one thousand series and decompose each down-sampled GST series. We then obtain the means of the multidecadal variability and of the trend and their spreads (uncertainty) from these decompositions. The results, which will be displayed later, show that the data end effect is minimal and is well within the estimated uncertainty bounds when GST time series is shortened by decades.

It should be noted that this new approach is motivated by Wu et al. (2007) and Huang et al. (2009b), where it was shown that the time series formed by summing the components of the yearly mean GST (resulting from EMD decomposition) with timescales shorter than two decades resembles white noise. The result can be confirmed using 
Fourier filtering instead of EMD decomposition. As demonstrated in previous studies (Huang and $\mathrm{Wu}$ 2008; Wu and Huang 2009), the low frequency components of data resulting from EEMD are not sensitive to temporally local perturbations, which implies that the randomly sampled monthly mean GST data for successive calendar years should contain almost the same MDV and GST signals as the annual mean GST time series if the data end effect and noise inherent in GST are small.

\section{ST and MDV in GST}

\subsection{ST and MDV of GST and their instantaneous rates of change}

In Wu et al. (2007) we applied EMD to the time series of annual values of GST in order to illustrate how the method works. In Fig. 2 we use EEMD to decompose global monthly land and sea surface temperature time series derived from HadCRUT3v dataset, which cover the period of record January 1850 through December 2008. Land temperature evidently exhibits greater variability than ocean temperature in the high frequency components $\mathrm{C} 1$, $\mathrm{C} 2$, and $\mathrm{C} 3$. When these are filtered out, the land and ocean time series become more similar, as evidenced by the consistently high positive correlations between respective modes obtained from the two decompositions (Table 1). Thompson et al. (2010) also show evidence of strong coherence between land and ocean temperature time series on these time scales. The features of interest in this study relate to the bottom two curves, which refer to $\mathrm{C} 8$ and $\mathrm{C} 9$ in the expansion. The bottom curves are $\mathrm{C} 9$ alone and the curves just above them are the sum of $\mathrm{C} 8$ and C9. Subsequent results discussed in this paper are based on an analogous EEMD analysis of the global-mean surface temperature (GST), combined land and ocean surface temperature time series.

In "Appendix" MDV and ST of GST (based on HadCRUT3v), as defined by $\mathrm{C} 8$ and C9, respectively, are shown to be distinguishable from a univariate red noise at above the $99 \%$ confidence level, and are in this sense statistically significant with reference to a univariate red noise null hypothesis. Figure 3 shows time series of ST and ST + MDV, all based on EEMD of the GST time series with land and ocean data combined, superimposed on the GST time series itself. ST exhibits continuous warming from 1850 to present, with a cumulative temperature rise of $0.75^{\circ} \mathrm{C}$, and inclusion of the MDV captures the stepwise character of the GST time series.

To assess the sensitivity of the estimated trends to the noise contained in GST and to the end point of the analysis, we use the down-sampling approach that was discussed in
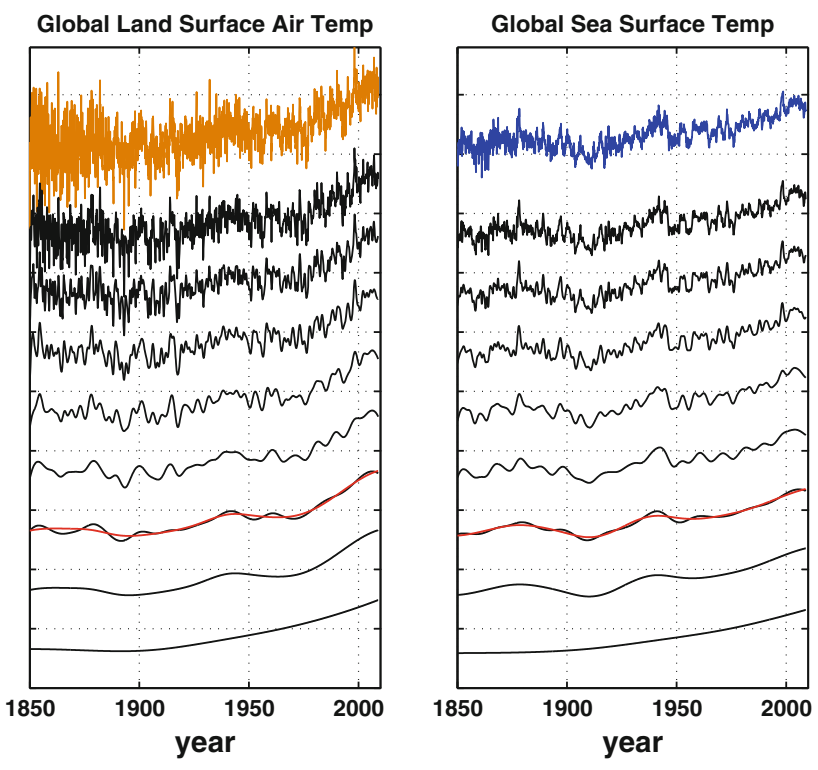

Fig. 2 EEMD decompositions of the time series of global-mean land surface air temperature (left panel), and of the global-mean sea surface temperature (right panel). In each panel, the top colored curve shows the raw time series; and the next eight black curves display successively the remainder time series after one more EEMD oscillatory mode is removed. The scales for all the curves are the same with the distance between the neighboring ticks being $1^{\circ} \mathrm{C}$. The red curves are identical to the black curves immediately below and is plotted to show how a remainder of data serves as a natural reference for the riding waves of higher frequency

Sect. 2.3: we estimated ST and MDV based on the period of record 1850-1949, 1850-1950, ..., 1850-2008 to obtain the 60 different estimates. Results of period of record 1850-1983, 1850-1988, 1850-1993, 1850-1998, 18502003, and 1850-2008 are shown in Figs. 4 and 5. Estimates of ST (C9) and MDV (C8) for the years prior to the 1940s are relatively insensitive to the end point, but the sensitivity is noticeably larger toward the end of the data records, as discussed in (Wu and Huang 2009). However, almost all the means of STs and of MDVs obtained based on any of these periods stay within the spreads of year 2008 (2 standard deviations) of the STs and MDVs calculated based on the down-sampled yearly time series for the period 1850-2008. In this sense, the MDV curve and the overall shape of the ST curve are robust with respect to changes in the end point despite the uncertainties toward the end of the record.

The contributions of ST and MDV to the linear trends in GST over the past 150, 100, 50, and 25 years are compared in Table 2. Notice that in the table, the linear trends in Figure TS. 6 of AR4 are based on the observed GST time series ending in 2003 while our calculation is based on the same GST time series ending in 2008. The last 5 years of relatively flat GST from 1998 onward leads to relatively smaller mean trends of the last 25 years and the last 
Table 1 Correlations between corresponding components of SST and SAT

\begin{tabular}{lllllllll}
\hline & $\mathrm{C}_{1}$ & $\mathrm{C}_{2}$ & $\mathrm{C}_{3}$ & $\mathrm{C}_{4}$ & $\mathrm{C}_{5}$ & $\mathrm{C}_{6}$ & $\mathrm{C}_{7}$ & $\mathrm{C}_{8}$ \\
\hline SST\&SAT & 0.08 & 0.21 & 0.26 & 0.45 & 0.70 & 0.61 & 0.70 & 0.57
\end{tabular}

SST the mean surface temperature over the oceans, SAT the mean surface air temperature over the land

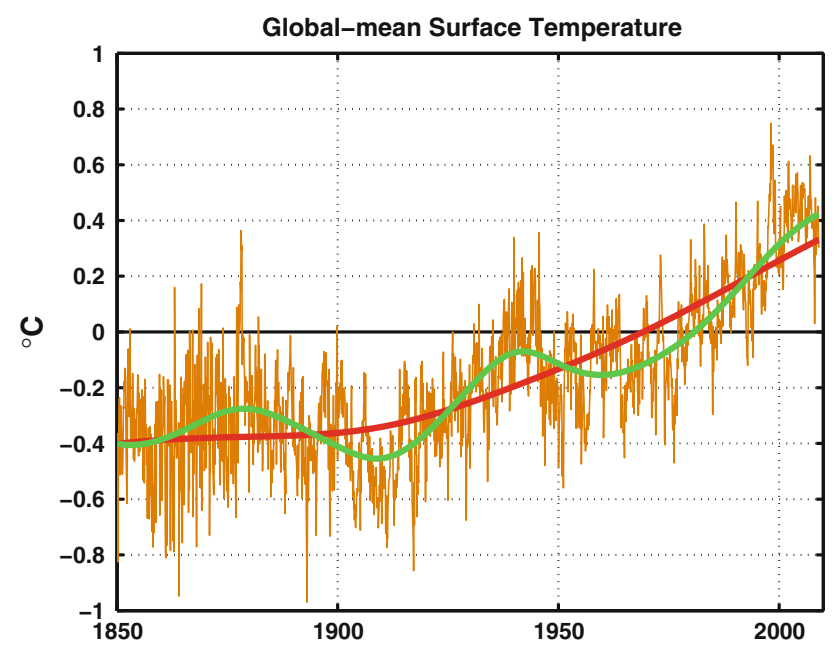

Fig. 3 Reconstruction of the raw GST time series (brown lines) using ST only (red lines) and ST + MDV (green lines)
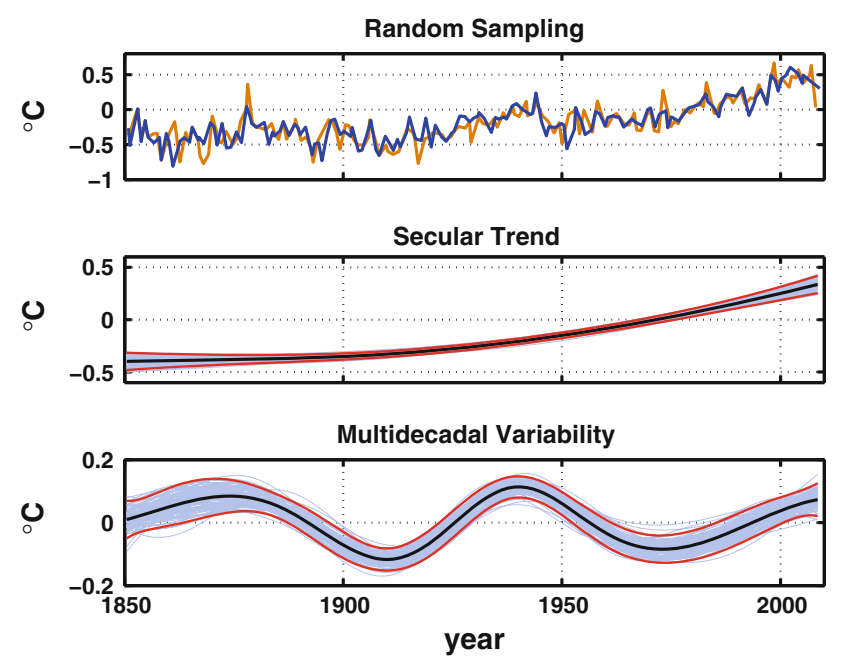

Fig. 4 STs and MDVs of 1,000 randomly sampled yearly GSTs. The top panel displays two randomly sampled yearly GSTs. In the mid panel, the silver lines are STs obtained from 1,000 randomly sampled yearly GSTs; the black line is the mean ST of all the 1,000 STs; and the red lines provide the yearly confidence intervals (within two standard deviations) for the 1,000 STs. The bottom panel is the same as the mid panel but for MDVs
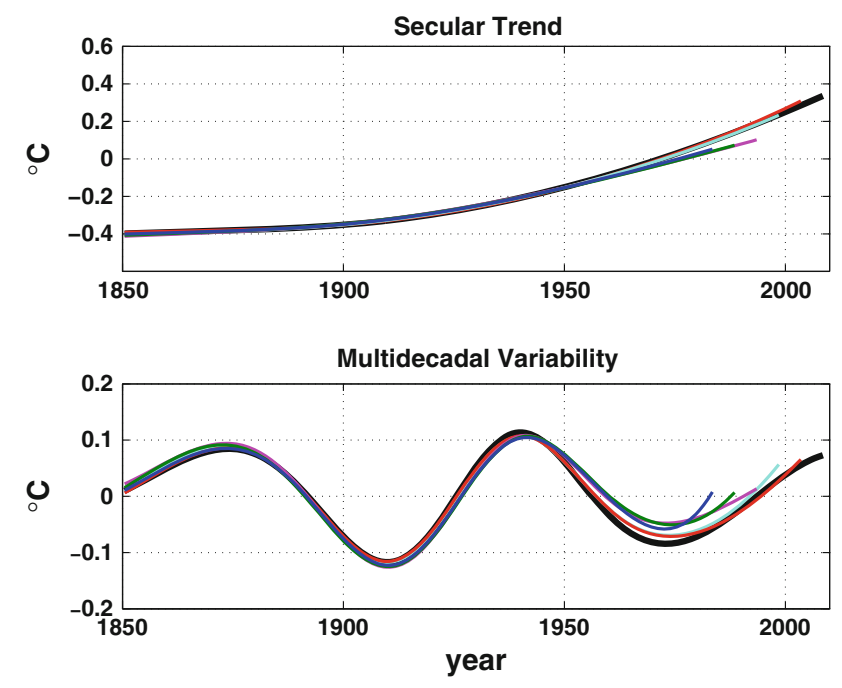

Fig. 5 Sensitivity of the EEMD-determined ST (upper panel) and MDV (lower panel) to the end date of GST data. In the upper (lower) panel, the blue, green, magenta, cyan, red, and black lines are the mean STs (MDVs) calculated based on the randomly sampled yearly GSTs ending in 1983, 1988, 1993, 1998, 2003, and 2008, respectively

50 years in our results than in AR4. On all time scales, the trends based on the time series formed by superimposing ST and MDV (i.e., the green curve in the upper panel of Fig. 3) are in close agreement with the trends based on the raw time series, consistent with the results that the high frequency components of yearly-averaged GST resemble white noise (Huang et al. 2009b). ST alone accounts for $0.43 \mathrm{~K}$ of the $0.53 \mathrm{~K}$ temperature increase over the past 50 years, consistent with the statement in the Summary for Policymakers in AR4 that "The observed changes ... support the conclusion that it is extremely unlikely that the global change of the past 50 years can be explained without external forcing and that it is very likely that it is not due to known natural causes alone". The estimated warming rate corresponding to the sum of ST and MDV over the past 25 years is $0.15 \pm 0.05 \mathrm{~K}$ per decade, of which $0.10 \pm 0.02 \mathrm{~K}$ per decade of warming is associated with ST only. The shape of ST closely parallels the global annual $\mathrm{CO}_{2}$ input to the atmosphere by the fossil fuel burning (figure not shown). Therefore, the estimated global warming due to human activities over the past 25 years ranges from about $0.10 \mathrm{~K}$ to about $0.15 \mathrm{~K}$ per decade, depending on the assumed partitioning of the MDV between natural and anthropogenic aerosol-forced variability: if variations in the circulation of the Atlantic Ocean play a prominent role in causing MDV, then the value should lie toward the lower end of this range. On the other hand, if a slowdown or reversal in the buildup of aerosols was primarily responsible for the increased rate of global warming toward the end of the twentieth century, then the 
Table 2 Mean slopes $\left({ }^{\circ} \mathrm{C} /\right.$ decade) of trends over different temporal spans

\begin{tabular}{lllll}
\hline & Last 150 years & Last 100 years & Last 50 years & Last 25 years \\
\hline AR4 & $0.045 \pm 0.012$ & $0.074 \pm 0.018$ & $0.128 \pm 0.026$ & $0.177 \pm 0.052$ \\
ST and MDV & $0.051 \pm 0.040$ & $0.086 \pm 0.039$ & $0.105 \pm 0.041$ & $0.148 \pm 0.051$ \\
ST & $0.050 \pm 0.014$ & $0.067 \pm 0.014$ & $0.086 \pm 0.018$ & $0.096 \pm 0.024$ \\
\hline
\end{tabular}

$A R 4$ the Fourth Assessment Report of the IPCC, $S T$ secular trend, MDV multidecadal variability as derived from EEMD analysis of GST with land and ocean data combined

human contribution should lie closer to the top end of the range.

The time derivative of ST, indicated by the red curve in Fig. 1, provides an indication of the rate at which global warming induced by the buildup of greenhouse gases and long-term aerosol change in the atmosphere has been proceeding, irrespective of the MDV associated with variations in the oceanic circulation and the relatively short-term changes in the rate of emission of aerosols. It is evident from the red curve in Fig. 1 that this rate has been increasing with time. The instantaneous warming rate is largest at the end of GST with a value of $0.10 \pm 0.03 \mathrm{~K}$ per decade. The instantaneous warming rates of ST calculated based on different periods of record of GST all stay within $\pm 0.03 \mathrm{~K}$ per decade of the warming rate of the mean ST calculated based on GST for 1850-2008.

The time derivative of ST + MDV, indicated by the blue curve in Fig. 1, replicates the step-like character of the 25 -year running mean trends (the black curve) and it also captures the recent slowdown in the rate of warming.

\subsection{Robustness of MDV and ST}

In this section, we examine the robustness of the results, with emphasis on the sensitivity of MDV and ST with respect to (a) a spurious discontinuity in the GST time series in 1945, (b) the inclusion or removal of the cool episodes following major volcanic eruptions, and (c) the presence of noise in the GST time series. All these calculations use the down-sampling approach described in Sect. 2.3.

\subsubsection{Effect of the spurious temperature discontinuity in 1945}

A spurious temperature drop in the GST time series derived from HadCRUT3v data, with an amplitude of about $0.3^{\circ} \mathrm{C}$ occurs starting in August 1945, when the U.S. Naval fleet, which was measuring sea surface temperature (SST) using thermometers embedded in the condenser intake returned to port and British ships, which were taking bucket measurements of SST, replaced them as the dominant source of SST data (Thompson et al. 2008, 2009). This problem was discovered in 2008; efforts are underway to correct it, but as of this time, it is known only that the correction required to eliminate the biases associated with the use of different measurement methods aboard ships operated by different nations will be negative prior to 1945 and positive thereafter and that these corrections will probably extend over one to as much as a few decades (Thompson et al. 2008).

It has been previously demonstrated that EEMD is a temporal local analysis method. If the biases are restricted to a relatively short time span, for example, less than a decade, the extracted MDV and ST should not be noticeably affected. If the duration is longer, e.g., a few decades, it is anticipated that there will be some phase shift in the MDV. Here, we consider the impact of a hypothetical, synthetic correction for this discontinuity generated by adding an exponential decaying function with an amplitude of $0.15^{\circ} \mathrm{C}$, ending in August 1945 and exponentially decaying backward in time and subtracting an exponential function with an amplitude of $0.15^{\circ} \mathrm{C}$ beginning in September 1945 and exponentially decaying forward in time. The $e$-folding time for both exponential functions is 15 years. The original GST, the GST correction function, and corrected GST are displayed in the top panel of Fig. 6.

It is clear from the bottom two panels of Fig. 6 that the MDVs of the original and "corrected" GST exhibit some differences between 1925 and 1975. The peak in the 1940s occurs a few years earlier in the "corrected" data and the minimum that appears in the original data in the 1960s is slightly shallower and shifted forward in time by about 10 years. The effect of the correction on the ST is barely discernible. Hence, unless the forthcoming real correction to the GST time series extends over time intervals substantially longer than assumed in designing this synthetic correction, it is not likely to qualitatively affect the results presented in this paper.

\subsubsection{Inclusion or exclusion of the response to volcanic eruptions}

Sulfur injected into the stratosphere by volcanic eruptions, condenses, forming long-lived layers of sulfate aerosols that reduce the shortwave solar radiation reaching the 


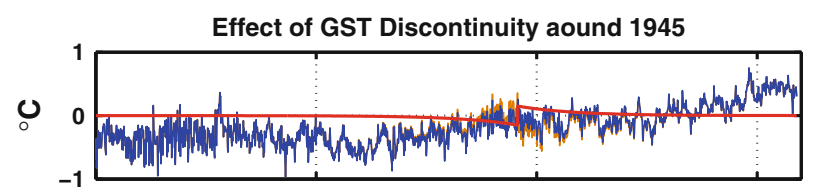

ST
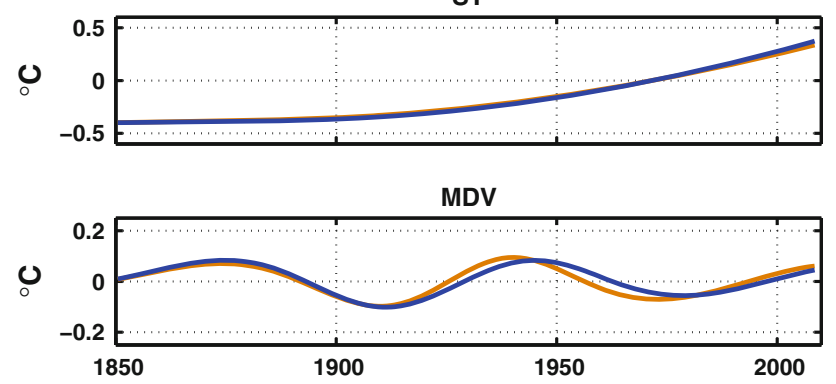

Fig. 6 EEMD decompositions of GST. The top panel shows the original GST data derived from HadCRUTv3 (brown line), a "corrected" version (blue line), and the correction function (red line). The bottom two panels show the STs and MDVs derived from the original and "corrected" GST using the same color convention. For this calculation, the downsampling approach has been applied; only the mean yearly STs (MDVs) are displayed in the middle (bottom) panel

Earth's surface. Because of the thermal inertia of the oceans, the resulting cooling of GST persists much longer than the aerosol layers that produce it. Since the volcanic eruptions occur intermittently throughout the GST record and the cool episodes following major eruptions in low latitudes can persist for up to 5-10 years, it is conceivable that volcanic forcing could affect the estimated MDV and ST.

To infer quantitatively how much this episodic volcanic forcing affects the estimated MDV and ST time series, we decompose the GST time series with the surface temperature response to the volcanic forcing removed. For this purpose we use the reconstruction of Thompson et al. (2009), in which the signatures of major low latitude volcanic eruptions of Santa Maria (1902), Agung (1963), El Chichon (1982), and Pinatubo (1991) are most clearly discernible, as indicated by the red line of the top panel of Fig. 7. The analysis is restricted for the period from 1900 onward, for which the volcanic forcing is best defined.

From the results shown in Fig. 7, it is evident that the removal of the response to volcanic eruptions in the GST time series has very little effect on the estimated ST. However, the effect on the MDV is quite significant: when the response to volcanic eruptions is removed, the MDV exhibits a pronounced peak around the year 2000 , with a rapid dropoff after that time that is not present in the original GST time series. But regardless of whether the response to volcanic eruptions is included or excluded, MDV exhibits a pronounced warming trend throughout most of the 1970s, the 1980s and the 1990s.

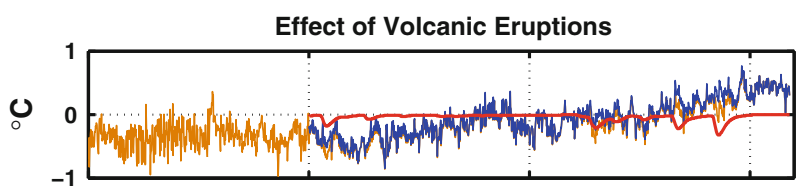

ST

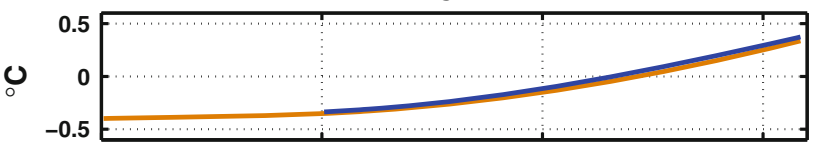

MDV

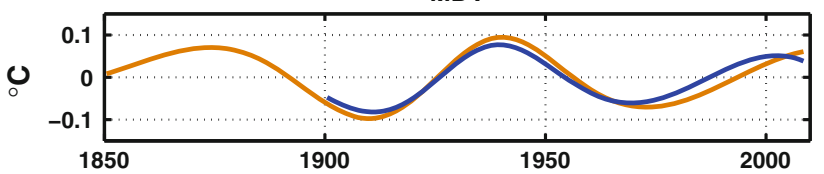

Fig. 7 Top panel: the original GST data derived from HadCRUTv3 (brown line), GST with the response to volcanic eruptions removed (blue line), and the surface temperature response to volcanic forcing (red line). Bottom two panels: ST and MDV of the GST, as computed including and excluding the effects of volcanic eruptions, plotted using the same color convention. For this calculation, the downsampling approach has been applied; only the mean yearly STs (MDVs) are displayed in the middle (bottom) panel

\subsubsection{The choice of dataset}

Although various versions of HadCRUT (Jones et al. 1999; Rayner et al. 2003) are the most widely used surface temperature analysis, there are other analyses, such as those provided by Goddard Institute for Space Studies (GISTEMP) (Hansen et al. 2009), and by the NOAA National Climate Data Center (Smith et al. 2008). Since the different analyses have used different methods to homogenize the observed surface air temperature and sea surface temperature observations, each of these products is slightly different. For example, 1998 is the warmest year based on HadCRUT, while in GISTEMP, 2005 is as warm as 1998. Furthermore, each of these analyses contains a different level of noise. Since there is not enough information to assess which of these products is the most accurate, we will restrict ourselves to assessing whether the results obtained by performing EEMD on the GST time series are sensitive to the choice of dataset.

In Fig. 8, we compare the MDV and ST modes derived from the GST and GISTEMP data. Since the GISTEMP is with respect to the mean annual cycle over the 30-year period 1950-1980 and the GST represents departures from the later (and warmer) 1960-1990 climatology, GISTEMP is systematically warmer than GST. This difference is reflected by the absolute value difference between the STs based in the two different analysis products at any given time. However, the STs closely parallel one another from 1950 onward, implying that the estimated trends in that time frame are almost identical. The major difference in the 


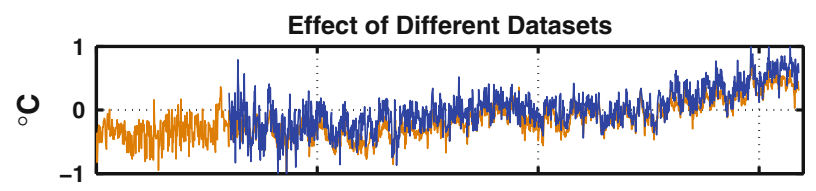

ST
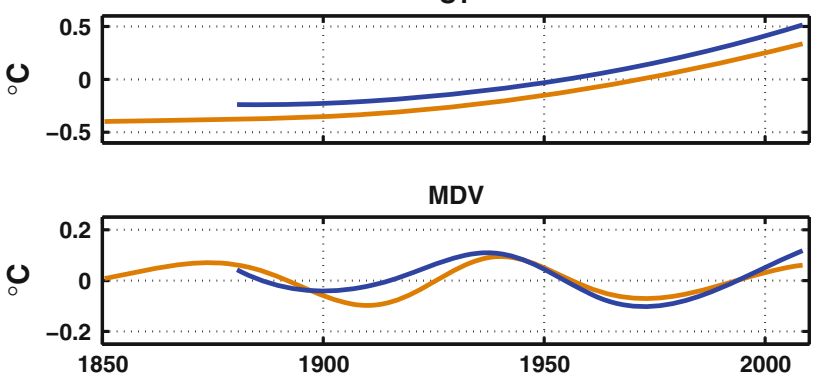

Fig. 8 Top panel: Monthly time series of GST (brown lines) and GISTEMP (blue lines). Bottom panels: ST and MDV modes derived from EEMD decompositions of the GST and GISTEMP time series plotted using the same color convention. For this calculation, the downsampling approach has been applied; only the mean yearly STs (MDVs) are displayed in the middle (bottom) panel

results is in the extracted MDVs in the early part of the record in which the data coverage was limited. From 1930 onward the two ST curves are quite similar. The raw time series and both the MDV and ST time series derived from GISTEMP exhibit slightly larger upward trends in the last decade or two of the record.

In the sensitivity experiments described in this section, we have demonstrated that the secular trend (ST or C9) mode recovered from EEMD is robust with respect to several prescribed perturbations in the input time series. The multidecadal variability (MDV or C8) mode exhibits some sensitivity with respect to the timing of the extrema, but the character of the variations is qualitatively similar in all cases and, in particular, all variants of the analysis exhibit a strong upward temperature trend in the late twentieth century that is reflected in both MDV and ST.

\subsection{Spatial structures of ST and MDV}

To get some indication of the physical processes that might be contributing to the ST and MDV in GST, we regressed the global surface air temperature (SAT) and global sea surface temperature (SST) fields onto the ST and MDV time series derived from EEMD of global (land plus ocean) surface temperature. The SAT dataset, which covers a time span from 1900 to December 2006, is from the Global Historical Climatology Network, version 3 (Peterson and Vose 1997), and the SST dataset is the NOAA ERSST by Smith et al. (2008), which spans the period or record January 1880 to December 2006. Regression maps for the periods from January 1900 (SAT) to December 2006 are shown in Fig. 9. Consistent with the linear trend maps in
Fig. 3.9 in AR4 (IPCC 2007) and the forced response and unforced internal variability over the most of global oceans of Ting et al. (2009), Knight (2009), and DelSole et al. (2011) the regression patterns for ST exhibit warming over the entire globe except for some spotty areas, e.g., the North Atlantic Ocean southeast of Greenland, southeastern United States and parts of China, where slight cooling has occurred. Such widespread warming is suggestive of a response to the buildup of well-mixed greenhouse gases, especially carbon dioxide. The close parallel between the ST curve and the carbon emission rate related to fossil fuel consumption after the industrial revolution also indicates that the buildup of atmospheric greenhouse gas concentrations projects almost exclusively onto ST, as does a substantial fraction of the buildup of aerosols injected into the atmosphere by human activities, activities, which can be assumed to be roughly linearly proportional to the rate of burning of fossil fuels (Crowley 2000). These results were confirmed by the analysis of CMIP3 model simulations by DelSole et al. 2011.

A noticeable feature of our regression pattern for MDV is that the dominant signals are restricted to high latitudes of the Northern Hemisphere and they appear to be more clearly defined over the ocean than over land (despite the fact that the amplitudes of surface temperature variation tend to be larger over land) and the sea surface temperature variations associated with MDV are particularly large over the Gulf Stream extension. These results are also consistent with the results of Semenov et al. (2010) which indicate that the North Atlantic-Arctic sector explains over $60 \%$ of the total Northern Hemisphere SAT response to surface flux anomalies of multidecadal timescale in their model experiments.

Both recent observational diagnoses (Zhang et al. 2007; Zhang 2008; Polyakov et al. 2009) and modeling evidence (Knight et al. 2005; Latif et al. 2006; Keenlyside et al. 2008; Semenov et al. 2010) suggest that variations in the intensity of the Atlantic meridional overturning circulation on the multidecadal time scale can give rise to episodes of rising and falling SST over the extratropical North Atlantic. In the SST pattern for the MDV shown in Fig. 9, the positive regression coefficients over the extratropical North Atlantic are accompanied by patches of negative coefficients over the Southern Ocean, a configuration reminiscent of the so-called "bi-polar seesaw" pattern inferred from paleoclimate proxies (Seidov and Maslin 2001; EPICA Community Members 2006), which is believed to be a consequence of variations in the strength of the Atlantic meridional overturning circulation. Another potential source of MDV that projects upon $\mathrm{C} 8$ derived from the EEMD analysis is the change in the Northern Hemisphere wintertime circulation that contributed to the rise in surface air temperature over the continents poleward 
Fig. 9 Global SAT and SST fields regressed onto the ST (upper panels) and MDV component of GST (lower panels). The left panels are for surface air temperature and the temperature. The gray areas indicate where the months with missing data are greater than $25 \%$ of the total months. Note that the scales on the color bars are different in the surface air temperature and sea surface temperature regression plots right panels are for sea surface
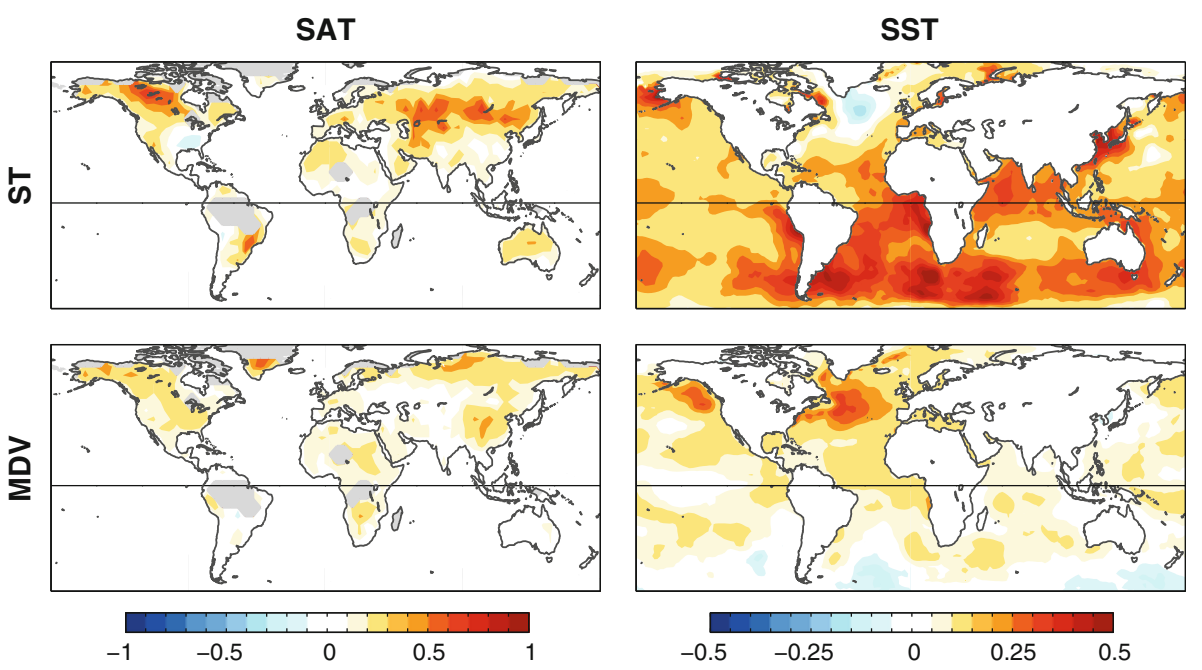

Fig. 10 Box averaged monthly mean SST time series (black lines) for the North Atlantic $\left(70^{\circ} \mathrm{W}-0^{\circ} \mathrm{E}\right.$, poleward of $\left.30^{\circ} \mathrm{N}\right)$ and the Southern Ocean (circumpolar, poleward of $45^{\circ} \mathrm{S}$ ), as indicated. The red lines indicate the secular trends (ST) as determined by EEMD, which serve as the reference lines for C8. The bottom panel shows the fraction of ICOADS data grid boxes with missing data in each month. See text for an explanation of the data sources

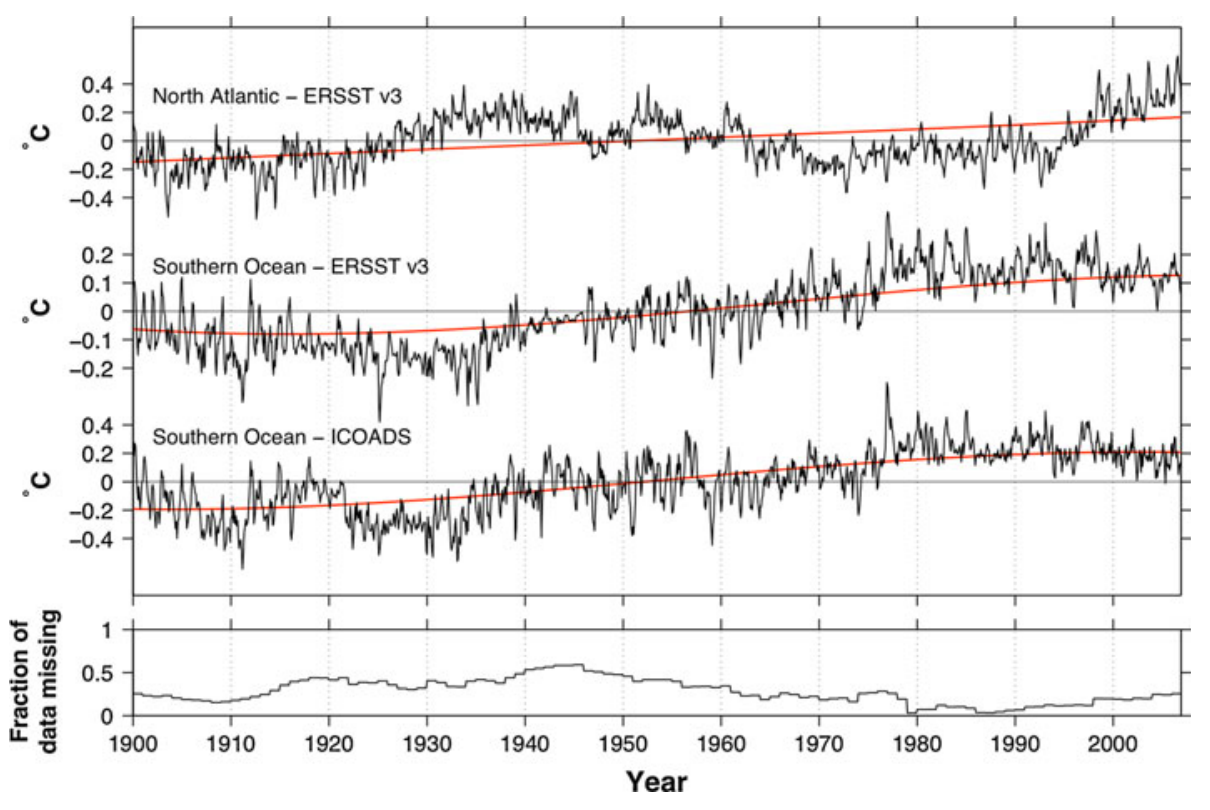

of $40^{\circ} \mathrm{N}$ during the late twentieth century (Wallace et al. 1995; Quadrelli and Wallace 2004, Fig. 16).

To substantiate that the patches of negative regression coefficients in Fig. 9 are really a reflection of an out-ofphase relationship between SST in the North Atlantic and Southern Ocean, we show in Fig. 10 time series of SST averaged over the two regions, as indicated in the caption. The North Atlantic time series and the topmost of the two Southern Ocean time series are based on data from ERSST. The bottom curve for the Southern Ocean is based on the International Comprehensive Ocean-Atmosphere Data Set (ICOADS), which contains objectively analyzed in-situ observations of SST in $5^{\circ} \times 5^{\circ}$ grid boxes (Smith and Reynolds 2005). When sampling is sparse within a grid box in a given month, the data are flagged as missing.
The two representations of the Southern Ocean time series closely parallel one another after 1930, during which time they exhibit a pronounced out-of-phase relationship with the North Atlantic time series on the multidecadal time scale. The correlation coefficient between C8 of the North Atlantic ERSST and the ERSST (ICOADS) representation of the Southern Hemisphere time series is $-0.77(-0.57)$. The length of these time series is not sufficient to establish the statistical significance of these multidecadal correlations, but at least it is evident that they are strong and the sign of them is consistent with the notion that the Atlantic multidecadal variability involves cross-equatorial heat fluxes associated with the thermohaline circulation, as demonstrated in Semenov et al. (2010). 
The results of a climate model simulations by Semenov et al. (2010), the statistical analysis of CMIP 3 forced and unforced runs by DelSole et al. (2011) and our analysis of observational data sets all points to the MDV being largely a reflection of internal variability of the climate system. However, the possibility that shorter term variations in aerosol forcing has contributed to the MDV cannot be ruled out. For example, the leveling off of sulfate concentrations around 1970 projects positively on that segment of the MDV curve (Murphy et al. 2009); indeed, it has been argued that the MDV in the second half of the twentieth century is dominated by this feature (Mann and Emanuel 2006).

\section{Summary and discussion}

In the previous sections, we have presented the results of EEMD analysis, which indicate that the secular warming trend during the 1980s and 1990s was not as large as the linear trends of the observation-based GST estimated in AR4 (IPCC 2007); and that the unprecedented rate of warming in the late twentieth century was a consequence of the concurrence of the upward swing of the multidecadal variability, quite possibly caused at least in part by an increase in the strength of the thermohaline circulation, and a secular warming trend due to the buildup of greenhouse gases. We estimate that as much as one third the warming of the past few decades as reported in Fig. TS.6 of the Summary for Policymakers of AR4 (IPCC 2007) may have been due to the speeding up of the thermohaline circulation. Other researchers have reached a similar conclusion: Keenlyside et al. (2008), Semenov et al. (2010) and DelSole et al. (2011) on the basis of numerical experiments with a climate model capable of representing the variability of the Atlantic meridional overturning circulation; Wild et al. (2007) on the basis of long term trends in the character of the diurnal temperature cycle at the Earth's surface; and Swanson et al. (2009) based on an analysis of the partitioning of the GST trends using linear discriminant analysis. Furthermore, by analyzing the temporal derivatives of ST, we have demonstrated that the secular warming trend in GST has not accelerated sharply in the past few decades.

In way of qualifications, we note that

1. The time derivative of ST of GST in the later twentieth century, as estimated by EEMD, is subject to future adjustments depending on how rapidly the atmosphere warms over the next decade or two.

2. The contribution of aerosol forcing to ST remains uncertain, as are the relative contributions of aerosol forcing and Atlantic MDV to the observed MDV of GST.
These caveats notwithstanding, the results presented here further substantiate the reality of human-induced global warming, as evidenced by the similarity between the secular trend curve recovered from EEMD of GST and the buildup of atmospheric greenhouse gas concentrations and by the near-global extent of the temperature increases associated with the secular trend. Our results also serve to highlight the importance of Atlantic multidecadal variability in mediating the rate of global warming, and they suggest that these variations deserve more explicit consideration in twentieth century climate simulations and in attribution studies based on recent observations of the rate of change of GST.

Acknowledgments The authors are benefited from the discussions with E. S. Sarachik, K.-K. Tung of U. Washington, E. K. Schneider of George Mason U., I. Fung of UC Berkeley, P. Chang of Texas A\&M $\mathrm{U}$, and R. Zhang of GFDL. NEH would like to acknowledge the support of a TSMC endowed chair at NCU, and also the support in part by a National Research Council of Taiwan grant NSC 95-2811M-008-027, and a (USA) Federal Highway Administration grant DTFH61-08-C-00028. ZW was sponsored by the NSF grants ATM0917743 and the First Year Asst Prof Award of Florida State University. JMW and BVS by NSF grant ATM-0812802. XC was supported by the National Basic Research Program of China 2007CB816002, National Science Foundation of China 40776018, and by National Key Technology R\&D Program 2006BAB18B02.

\section{Appendix: Robustness of MDV and ST with respect to superimposed noise}

DO the MDV and ST time series obtained in this study contain significant signals that can be distinguished from background noise? To address this question, we discuss first the general concept of statistical significance of a component of a time series extracted using a method such as EEMD .

In general, climate data can be represented by a combination of signal and noise,

$x(t)=s(t)+n(t)$,

where $x(t)$ represents the data and $s(t)$ and $n(t)$ refer to the signal and the noise, respectively. When a decomposition method is applied to $x(t)$ and a set of components is obtained, it is of interest to determine whether a specified component contains a signal. A definitive answer to this question is impossible, for we know a priori neither the signal $s(t)$ nor the noise $n(t)$. A less demanding and widely used approach is first to assume the characteristics of the background noise based on a preliminary understanding of the system for which the data are recorded, and then, for each component, to discern the part of $x(t)$ that stands out above this assumed noise at some specified confidence level. 
In this approach, there are two important issues: (1) the null hypothesis concerning the data, or the assumed characteristics of the noise inherent in the data; and (2) the metric (or metrics) to be used in discerning between the between the signal and the noise. With respect to the first issue, a widely used null hypothesis that is not without some physical justification is that climate system is stochastically forced by white (weather) noise (Hasselmann 1976; Barsugli and Battisti 1998; Schneider and Fan 2007), and therefore that the noise can be represented as a first order Markov process. With respect to the second issue, widely used metrics are the probability density function and the Fourier spectra of the noise. Protocols for testing statistical significance of components derived from Empirical Mode Decomposition (EMD) or Ensemble Empirical Mode Decomposition (EEMD) have not yet been fully developed. In Wu and Huang (2004) a statistical method that uses the temporal variance as a metric is used for assessing the statistical significance of a component, tested against white noise. However, this method is not applicable for a null hypothesis of noise other than white. Therefore, a more general approach for testing the statistical significance of a component derived from EEMD needs to be designed.

The details of this new method will be reported elsewhere. Here we present the general ideas behind this method for testing the significance of MDV and ST against a first order Markov process. EEMD decomposes data $x(t)$ in terms of a set of components from high frequency to low frequency $c_{j}, j=1, \ldots, n$, and a remainder $r_{n}$, which varies on timescales longer than the longest timescale of any oscillatory component $c_{j}$, i.e.,

$x(t)=\sum_{j=1}^{n} c_{j}+r_{n}=d_{n}+r_{n}$,

where $r_{n}$ has been referred to as the "trend" (Huang et al. 1998; Wu et al. 2007). The metric that we use in this approach is the autocorrelation function, $\rho_{n}(\tau)$, of the time series made up of the sum of the first $n$ oscillatory components, i.e.,

$\rho_{n}(\tau)=\frac{\sum_{i=1}^{N-\tau} d_{n}(i) d_{n}(i+\tau)}{\sqrt{\sum_{i=1}^{N-\tau} d_{n}^{2}(i)} \sqrt{\sum_{i=1}^{N-\tau} d_{n}^{2}(i+\tau)}}$.

In general, if the amplitude of $r_{n}$ is very small, the difference between $\rho_{n}(\tau)$ and $\rho_{n+1}(\tau)$ will also be very small, from which it follows that $r_{n}$ is not statistically significant. If the difference between $\rho_{n}(\tau)$ and $\rho_{n+1}(\tau)$ is large, it can be concluded that the contribution of $c_{n+1}$ to $\rho_{n+1}(\tau)$ is large, and $c_{n+1}$ must be of large amplitude, and therefore $c_{n+1}$ is statistically significant. Figure 11 shows $\rho_{n}(\tau)$ for global-mean surface temperature (GST) and for a time series generated using a first order Markov process with an autocorrelation of 0.88 at a lag of one data-point, which matches the autocorrelation of the GST time series. Clearly, the differences between $\rho_{7}(\tau), \rho_{8}(\tau)$, and $\rho_{8}(\tau)$ for the GST are quite dramatic. In comparison, the differences between the corresponding autocorrelation functions for the time series generated using a first order Markov process are much smaller. A Monte-Carlo test using 1,000 different realizations of time series following a first order Markov process with the same autocorrelation shows that there is no case in which the differences between $\rho_{7}(\tau)$ and $\rho_{8}(\tau)$, and $\rho_{8}(\tau)$ and $\rho_{9}(\tau)$ are nearly as large as those for GST. The results constitute proof that the MDV and ST are virtually unaffected by the presence of random noise in the higher frequency EEMD modes and thus can be regarded as distinct from stochastically forced climate variability driven by (weather) noise and in this sense statistically significant.

In the above, we have established the significance of the MDV and ST with respect to a univariate red noise null hypothesis. However, it has been demonstrated in some studies (e.g., Schneider and Cornuelle 2005; Chang et al. 2004; Newman 2007) that a univariate red noise null hypothesis may not be most appropriate. Other studies have suggested multivariate null hypothesis (Livezey and Smith 1999; Penland and Matrosova 2006). It remains to be investigated which null hypothesis is most appropriate for GST analyzed here.
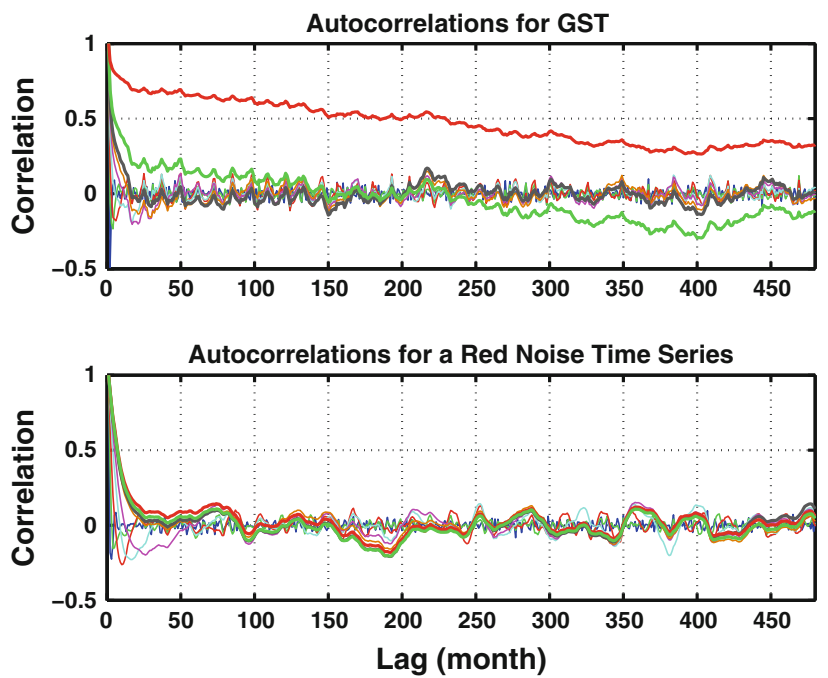

Fig. 11 Autocorrelation functions $\rho_{n}(\tau)$ for the sum of components 1 to $n$. The thin blue, green, red, cyan, magenta, and brown lines are for $\rho_{1}(\tau)-\rho_{6}(\tau)$, respectively; and the bold black, green, and red lines are for $\rho_{7}(\tau), \rho_{8}(\tau)$, and $\rho_{9}(\tau)$, respectively. The upper panel is for GST, and the lower panel is for a time series generated using the first order Markov process with the same one-month-lag autocorrelation as that of GST 


\section{References}

Barsugli JJ, Battisti DS (1998) The basic effects of atmosphere-ocean thermal coupling on midlatitude variability. J Atmos Sci 55:477493

Chang P, Saravanan R, Wang F, Ji L (2004) Predictability of linear coupled systems. Part II: an application to a simple model of tropical Atlantic variability. J Clim 17:1487-1503

Crowley TJ (2000) Causes of climate change over the past 1000 years. Science 289:270-277

Daubechies I (1992) Ten lectures on wavelets. Society for Industrial and Applied Mathematics, Philadelphia

DelSole T, Tippett MK, Shukla J (2011) A significant component of unforced multidecadal variability in twentieth century global warming. J Clim (in press)

EPICA Community Members (2006) One-to-one coupling of glacial climate variability in Greenland and Antarctica. Nature 444:195198

Fan J, Yao Q (2005) Nonlinear time series: nonparametric and parametric methods. Springer, New York

Flandrin P, Rilling G, Gonçalvès P (2004) Empirical mode decomposition as a filter bank. IEEE Signal Process Lett 11:112-114

Gabor D (1946) Theory of communication. J Inst Electr Eng 93:429_ 457

Gledhill RJ (2003) Methods for investigating conformational change in biomolecular simulations. A dissertation for the degree of Doctor of Philosophy at Department of Chemistry, the University of Southampton, $201 \mathrm{pp}$

Härdle W (1990) Applied nonparametric regression. Cambridge University Press, Cambridge

Hansen J, Ruedy R, Glascoe J, Sato M (1999) GISS analysis of surface temperature change. J Geophys Res 104:30997-31022

Hasselmann K (1976) Stochastic climate models, part I: theory. Tellus 28:473-485

Huang NE, Wu Z (2008) A review on Hilbert-Huang transform: the method and its applications on geophysical studies. Rev Geophys 46:RG2006. doi:10.1029/2007RG000228

Huang NE, Shen Z, Long SR, Wu MC, Shih EH, Zheng Q, Tung CC, Liu HH (1998) The empirical mode decomposition method and the Hilbert spectrum for non-stationary time series analysis. Proc R Soc Lond 454A:903-995

Huang NE, Shen Z, Long RS (1999) A new view of nonlinear water waves-the Hilbert spectrum. Annu Rev Fluid Mech 31:417457

Huang NE, Wu ML, Long SR, Shen SS, Qu WD, Gloersen P, Fan KL (2003) A confidence limit for the empirical mode decomposition and the Hilbert spectral analysis. Proc R Soc Lond 459A:23172345

Huang NE, Wu Z, Long SR, Arnold KC, Chen X, Blank K (2009a) On instantaneous frequency. Adv Adapt Data Anal 1:177-229

Huang NE, Wu Z, Pinzón JE, Parkinson CL, Long SR, Blank K, Gloersen P, Chen X (2009b) Reductions of noise and uncertainty in annual global surface temperature anomaly data. Adv Adapt Data Anal 1:447-460

IPCC (2007) Climate change 2007: the scientific basis. Contribution of Working Group I to the Third Assessment Report of the Intergovernmental Panel on Climate Change. Cambridge University Press, Cambridge

Jones PD, New M, Parker DE, Martin S, Rigor IG (1999) Surface air temperature and its changes over the past 150 years. Rev Geophys 37:173-199

Keenlyside NS, Latif M, Jungclaus J, Kornblueh L, Roeckner E (2008) Advancing decadal-scale climate prediction in the North Atlantic sector. Nature 453:84-88
Knight JT (2009) The Atlantic multidecadal oscillation inferred from the forced climate response in coupled general circulation models. J Clim 22:1610-1625

Knight JT, Allan RJ, Folland CK, Vellinga M, Mann ME (2005) A signature of persistent natural thermohaline circulation cycles in observed climate. Geophys Res Lett 32:L20708. doi:1029/2005 GL024233

Latif M, Collins M, Pohlmann H, Keenlyside N (2006) A review of predictability studies of the Atlantic sector climate on decadal time scales. J Clim 19:5971-5987

Livezey RE, Smith TM (1999) Covariability of aspects of North American climate with global sea surface temperatures on interannual to interdecadal timescales. J Clim 12:289-302

Mann ME, Emanuel KA (2006) Atlantic hurricane trends linked to climate change. EOS 87:233-244

Murphy DM, Solomon S, Portmann RW, Rosenlof KH, Forster PM, Wong T (2009) An observationally based energy balance for the Earth since 1950. J Geophys Res 114:D17107. doi:10.1029/ 2009JD012105

Newman Matthew (2007) Interannual to decadal predictability of tropical and North Pacific Sea surface temperatures. J Climate 20:2333-2356

Penland C, Matrosova L (2006) Studies of El Nino and interdecadal variability in tropical sea surface temperatures using a nonnormal filter. J Clim 19:5796-5815

Peterson TC, Vose RS (1997) An overview of the global historical climatology network temperature database. Bull Am Meteorol Soc 78:2837-2849

Polyakov IV, Alexeev VA, Bhatt US, Polyakova EI, Zhang X (2009) North Atlantic warming: patterns of long-term trend and multidecadal variability. Clim Dyn. doi:10.1007/s00382-008-0522-3

Quadrelli R, Wallace JM (2004) A simplified linear framework for interpreting patterns of Northern Hemisphere wintertime climate variability. J Clim 17:3728-3744

Rayner NA, Parker DE, Horton EB, Folland CK, Alexander LV, Rowell DP, Kent EC, Kaplan A (2003) Global analyses of sea surface temperature, sea ice, and night marine air temperature since the late nineteenth century. J Geophys Res 108:4407. doi: 10.1029/2002JD002670

Schneider N, Cornuelle BD (2005) The forcing of the pacific decadal oscillation. J Clim 18:4355-4373

Schneider EK, Fan M (2007) Weather noise forcing of surface climate variability. J Atmos Sci 64:3265-3280

Seidov D, Maslin M (2001) Atlantic Ocean hear piracy and the bipolar climate see-saw during Heinrich and Dansgaard-Oeschger events. J Quat Sci 16:321-328

Semenov VA, Latif M, Dommenget D, Keenlyside NS, Strehz A, Martin T, Park W (2010) The impact of North Atlantic-Arctic multidecadal variability on northern hemisphere surface air temperature. J Clim 23:5668-5677

Smith TM, Reynolds RW (2005) A global merged land air and sea surface temperature reconstruction based on historical observations (1880-1997). J Clim 18:2021-2036

Smith TM, Reynolds RW, Peterson TC, Lawrimore J (2008) Improvements to NOAA's historical merged land-ocean surface temperature analysis (1880-2006). J Clim 21:2283-2296

Stock JH, Watson MW (1988) Variable trends in economic time series. J. Econ Persp 2:147-174

Swanson KL, Sugihara G, Tsonis AA (2009) Long-term natural variability and 20th century climate change. Proc Natl Acad Sci USA 106:16120-16123

Thompson DWJ, Kennedy JJ, Wallace JM, Jones PD (2008) A large discontinuity in the mid-twentieth century in observed globalmean surface temperature. Nature 453(29):646-649. doi: $10.1038 /$ nature 06982 
Thompson DWJ, Wallace JM, Jones PD, Kennedy JJ (2009) Identifying signatures of natural climate variability in time series of global-mean surface temperature: methodology and insights. J Clim 22:6120-6141

Thompson DWJ, Wallace JM, Kennedy JJ, Jones PD (2010) An abrupt drop in Northern Hemisphere sea surface temperature around 1970. Nature 467:444-447. doi:10.1038/nature09394

Ting M, Kushnir Y, Seager R, Li C (2009) Forced and internal twentieth-century SST trends in the North Atlantic. J Clim 22:1469-1481

Torrence C, Compo GP (1998) A practical guide to wavelet analysis. Bull Am Meteorol Soc 79(1):61-78

Van der Pol B (1946) The fundamental principles of frequency modulation. Proc IEE 93:153-158

Wallace JM, Zhang Y, Renwick JA (1995) Dynamic contribution to hemispheric mean temperature trends. Science 270:780-783

Wild M, Ohmura A, MuKowski K (2007) Impact of global dimming and brightening on global warming. Geophys Res Lett 34:L04702. doi:10.1029/2006GL028031

Wu Z, Huang NE (2004) A study of the characteristics of white noise using the empirical mode decomposition method. Proc R S Lond 460A:1597-1611
Wu Z, Huang NE (2005) Statistical significant test of intrinsic mode functions. In: Huang NE, Shen SSP (eds) Hilbert-Huang transform: introduction and applications. World Scientific, Singapore, pp 125-148

Wu Z, Huang NE (2009) Ensemble empirical mode decomposition: a noise-assisted data analysis method. Adv Adapt Data Anal $1: 1-41$

Wu Z, Huang NE, Long SR, Peng C-K (2007) On the trend, detrending and variability of nonlinear and non-stationary time series. Proc Natl Acad Sci USA 104:14889-14894

Wu Z, Huang NE, Chen X (2009) The multi-dimensional ensemble empirical mode decomposition method. Adv Adapt Data Anal $1: 339-372$

Zhang R (2008) Coherent surface-subsurface fingerprint of the Atlantic meridional overturning circulation. Geophys Res Lett 35:L20705. doi:10.1029/2008GL035463

Zhang R, Delworth TL, Held IM (2007) Can the Atlantic Ocean drive the observed multidecadal variability in Northern Hemisphere mean temperature? Geophys Res Lett 34:L02709. doi: 10.1029/2006GL028683 